\title{
A new micro scale FE model of crystalline materials in micro forming process
}

\author{
Liang Luo ${ }^{1}$, Haibo Xie ${ }^{1}$, Dongbin Wei ${ }^{1,2}$, Xiaogang Wang ${ }^{3}$, Cunlong Zhou ${ }^{3}$ and Zhengyi Jiang ${ }^{1, \text { a }}$ \\ ${ }^{1}$ School of Mechanical, Materials \& Mechatronic Engineering, University of Wollongong, Wollongong NSW 2500, Australia \\ ${ }^{2}$ School of Electrical, Mechanical and Mechatronic Systems, University of Technology, Sydney, NSW 2007, Australia \\ ${ }^{3}$ School of Material Science and Engineering, Taiyuan University of Science and Technology, Taiyuan, Shanxi 030024, China
}

\begin{abstract}
Micro forming of metals has drawn global attention due to the increasing requirement of micro metal products. However, the size effects become significant in micro forming processes and affect the application of finite element (FE) simulation of micro forming processes. Dividing samples into small areas according to their microstructures and assigning individual properties to each small area are a possible access to micro forming simulation considering material size effects. In this study, a new model that includes both grains and their boundaries was developed based on the observed microstructures of samples. The divided subareas in the model have exact shapes and sizes with real crystals on the sample, and each grain and grain boundaries have their own properties. Moreover, two modelling methods using different information from the microstructural images were introduced in detail. The two modelling methods largely increase the availability of various microstructural images. The new model provides accurate results which present the size effects well.
\end{abstract}

\section{Introduction}

Micro manufacturing has drawn global attention due to the increasing requirement on the micro products. Once scaled down to a micro scale, significant size effects on the micro fabrication processes and on the micro products become considerable [1-3]. Individual grain impacts on deformation behaviour substantially and causes great data scatter in experiments. Therefore, the finite element (FE) simulation, which generally cannot reflect these phenomena, needs to consider the size effects. The modified Swift and Hall-Petch (H-P) models were introduced to present strain-stress relationships on the micro scale $[4,5]$. Thus, simulation using these accurate micro scale strain-stress relationships can provide acceptable results. Additionally, the surface layer model and the modified surface layer model were adopted to explain the decrease of flow stress with decreased sample sizes [6, 7]. However, all the aforementioned models cannot explain the increasing data scatter from experiments and the localised deformation behaviour caused by the individual grain. To further reveal the size effects, the Voronoi models were developed [8-10]. The sample was divided into small subareas according to the Voronoi structures and the small subareas were assigned different mechanical properties. Consequently, the size effects, such as the decrease of the flow stress and the locally special deformation, can be expressed by the distribution of mechanical properties on the subareas. Furthermore, the centroidal Voronoi structure with similar Voronoi cells is comparable to the annealed metals with equiaxed crystals. Thus the Voronoi models can present the sample on a grain level. Nevertheless, not every metal has the equiaxed crystalline microstructure and the equiaxed crystals are not exactly the same as the centroidal Voronoi cells. Although the distribution and sizes of the Voronoi cells can be adjusted by a density function, the cells distribution can hardly match the real microstructures of the samples. Due to a large quantity of grains on the sample, the grains are generally classified into several groups, and each group has one kind of mechanical properties. As there are no clear classification criteria, all the Voronoi cells are organised randomly. Grain boundaries, that play an important role in the micro forming process, are generally ignored in the Voronoi simulation models. Thus, the Voronoi models are not accurate enough compared to the real micro samples. To precisely account for the size effects, a new micro scale model is highly required. The subareas on the new model should have exact shapes and sizes to the crystals on the sample. The grains' arrangement is based on reasonable classification rules, such as the actual grains sizes or grains geometrical orientation. The grains and grain boundaries should be considered separately.

In this study, a real microstructure simulation model considering grains and their boundaries separately was developed by two different modelling methods. The two modelling methods were explained and the arrangement of grains was discussed. Next, the developed models were utilised in the micro deep drawing simulation.

\footnotetext{
Corresponding author: jiang@uow.edu.au
} 
Finally, simulation results were discussed and compared with experimental results.

\section{Modelling Method I}

The first modelling method uses the microstructural images with clear grain boundaries from various instruments, such as metallurgic microscope and electron backscatter diffraction (EBSD). The grains were detected and then all the grain boundaries were identified through an image processing program in MATLAB. Fig. 1(a) shows a typical microstructure image from the EBSD. The microstructural image was firstly read into MATLAB and then transferred into a binary image where there are only black grain boundaries and white grains. During the transformation, noise and small holes were eliminated. Therefore, non-grain information on the image was omitted. Subsequently, the binary image was treated with the Moore-Neighbour tracing algorithm modified by Jacob's stopping criteria. All the grains, the individual closed subareas, were then detected and sorted, as shown in Fig. 1(b), and their information was stored in
MATLAB. During the detection, each grain's area, geometrical centre and geometrical orientation can be calculated. The blue ports on Fig. 1(b) are the grains geometrical centres.

Simultaneously, a normal finite element model was generated where all the elements were in a single part with the same mechanical properties. The elemental and nodal information was then imported into MATLAB. The centre of each element was used to present the elemental positon. Then, the elements were mapped onto the grains detected by MATLAB. By comparing all the grains and the elements positions, the elements inside one grain were assigned the corresponding grain's ID number. Accordingly, the elements in one grain were set as one individual part with separate mechanical properties. By contrast, the elements that belong to none of the gains were defined as the grain boundaries. Finally, the updated relating elemental information, part and material properties indexes, were written into the pre-built normal finite element model. Fig. 1(c) displays the final finite element model based on the microstructure as shown in Fig. 1(a).

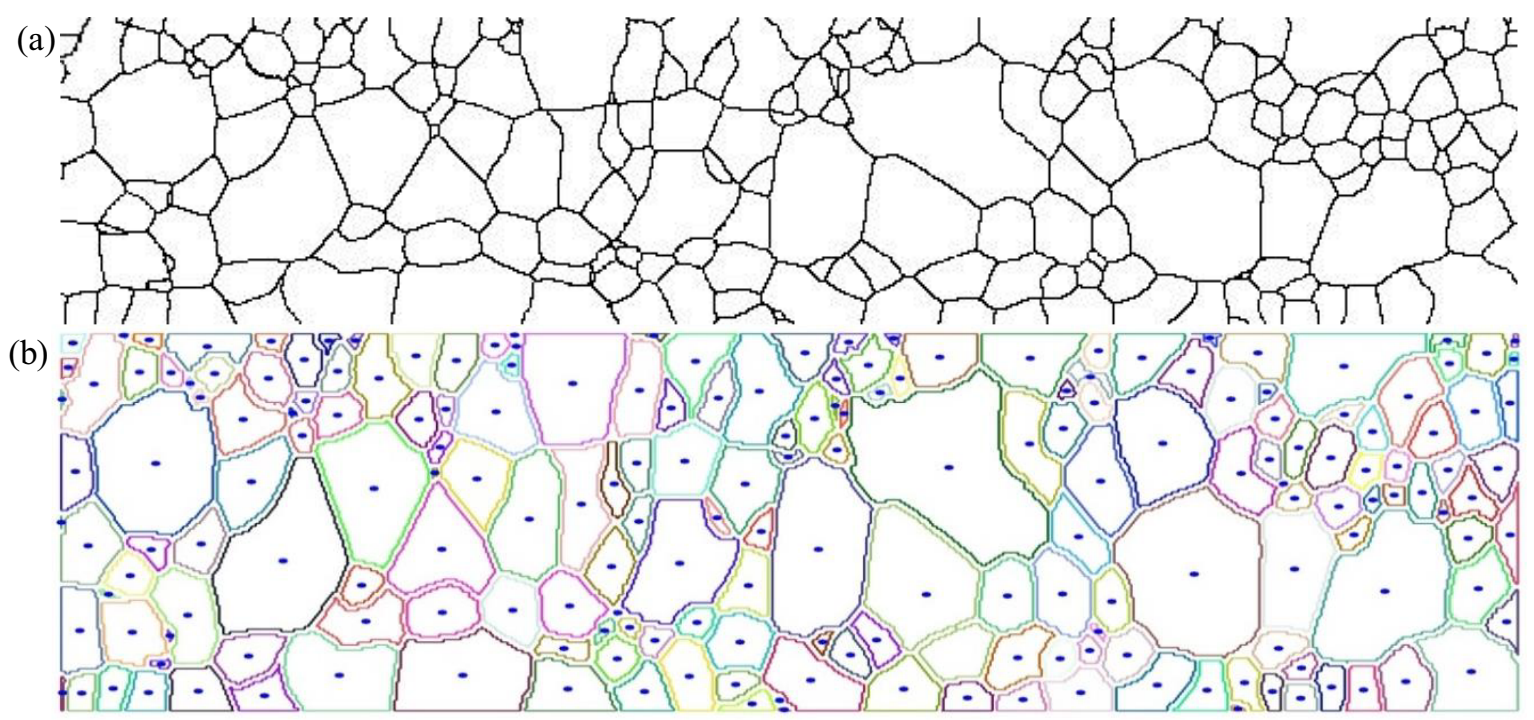

(c)

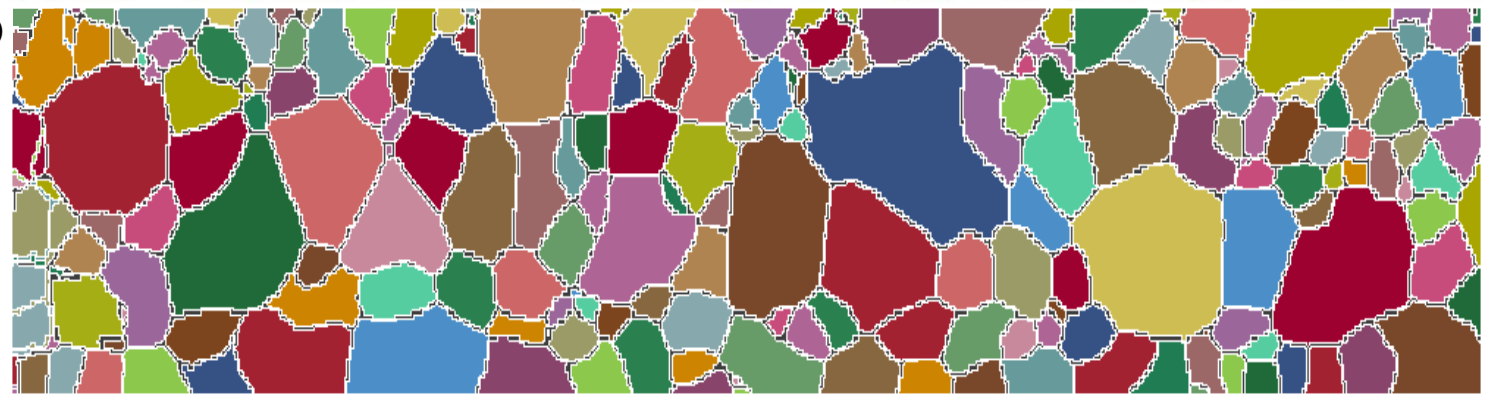

Figure 1. (a) Microstructure of a sample from EBSD, (b) its corresponding geometry detected by MATLAB and (c) corresponding simulation model.

As the simulation model is on the micro scale, there are a great quantity of grains and their accordant mechanical properties in the simulation model. Thus, the simulation model can be very large and over the executable size of the commercial simulation software. Therefore, all the grains should be categorised as several groups, and each group has one type of mechanical properties. The overall simulation model size can then be greatly reduced due to the limited information about material properties. In the aforementioned Voronoi models, the grains were classified randomly or based on the grains' statistic information. By contrast, the grains areas and their geometrical orientations can be utilised for reasonable arrangement in the new model. Generally, samples with relatively large grains have relatively low flow resistance and low tensile strength. Accordingly, large grains were set low mechanical properties, while small grains were assigned high mechanical properties. 
Furthermore, the grain boundaries were allocated mean mechanical properties of all grain groups. Fig. 2(a) shows the grain area distribution of the sample illustrated in Fig. 1(a), and Fig. 2(c) displays the corresponding finite element simulation model. Five colours indicated five area levels of grains and five grain groups. Regarding grain orientation, although the orientation is calculated based on grain's geometry, this geometry indicates the former processing of the material and thus the preferred deformation direction of the grain. The geometrical orientation is the angle (in degrees ranging from 0 to 180 degrees) between the $\mathrm{x}$-axis (horizontal axis) and the major axis of the ellipse that has the same secondmoments as the detected grain. Thus, all the grains orientations were analysed and all the grains were classified into several groups accordingly. In addition, the anisotropic material model can be utilised and relating parameters indicating anisotropy were set according to the orientation angle. For example, the in-plane anisotropic value, the $R$ value defined as Eq. (1), is generally used for presentation of material anisotropy. At any angle $\emptyset, R_{\varnothing}$ can be calculated from Eq. (2). According to the 3-parameter Barlat yielding criteria, Eq. (3), anisotropic properties of the grains with different orientation angles can be considered. Fig. 2(b) presents the orientation distribution of the grains and Fig. 2(d)

(a)

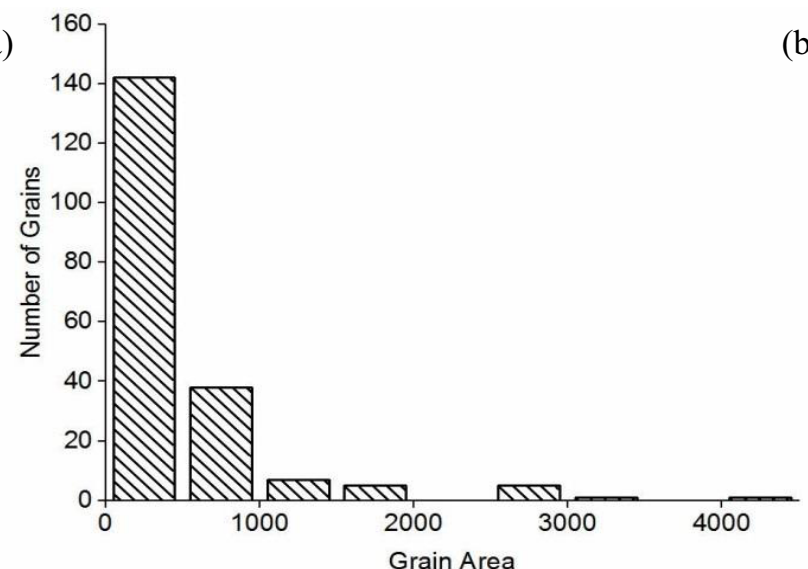

exhibits the corresponding simulation model.

$$
\begin{gathered}
R=\frac{\frac{d W}{d \varepsilon} / W}{\frac{d T}{d \varepsilon} / T} \\
R_{\emptyset}=\frac{2 m \sigma_{Y}^{m}}{\left(\frac{\partial \Phi}{\partial \sigma_{x}}+\frac{\partial \Phi}{\partial \sigma_{y}}\right) \sigma_{\emptyset}}-1 \\
2 \sigma_{Y}^{m}=a\left|K_{1}+K_{2}\right|^{m}+a\left|K_{1}-K_{2}\right|^{m}+c\left|2 K_{2}\right|^{m} \\
K_{1}=\frac{\sigma_{x}+h \sigma_{y}}{2} \\
K_{2}=\sqrt{\left(\frac{\sigma_{x}-h \sigma_{y}}{2}\right)^{2}+p^{2} \tau_{x y}^{2}}
\end{gathered}
$$

where $R$ is the in-plane anisotropy value, $W$ and $T$ are the width and thickness respectively, $\sigma_{\varnothing}$ is the uniaxial tension in the $\varnothing$ direction, $\sigma_{Y}$ is the yield stress, $m$ is 8 for face cantered cubic materials, $\sigma_{x}$ and $\sigma_{y}$ are the stress along $x$ and $y$ directions respectively, $\tau_{x y}$ is the shear stress, $K_{1}$ and $K_{2}$ are given by Eqs. (4) and (5) respectively, $a, c, h$ and $p$ are the orientation related material parameters and for isotropic material $a=c=h=p=1$.

(b)

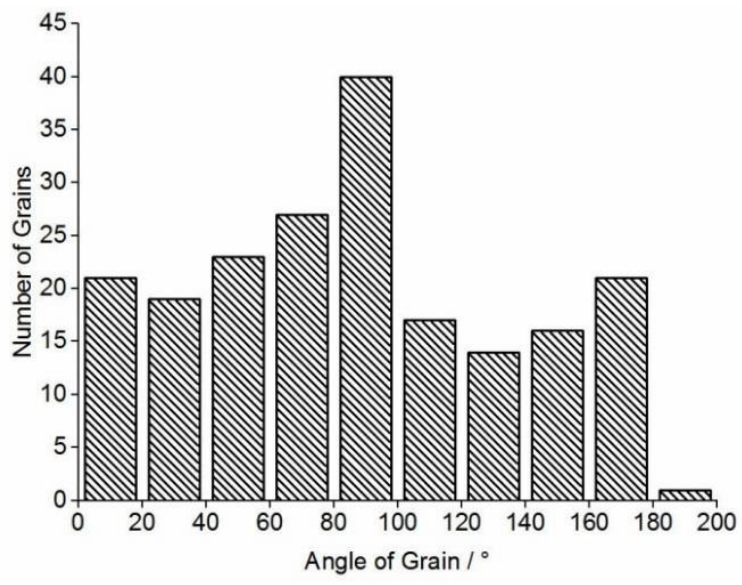

(c)

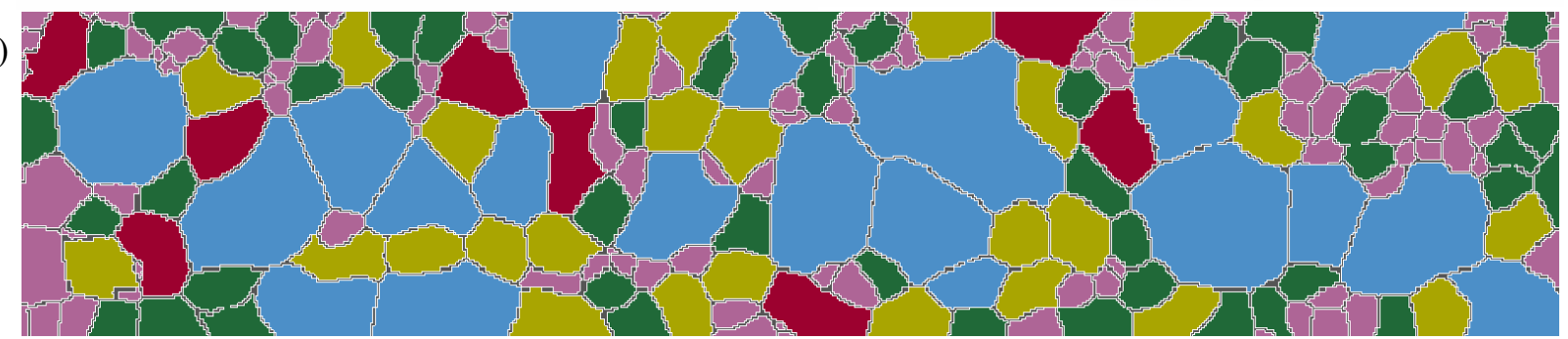

(d)

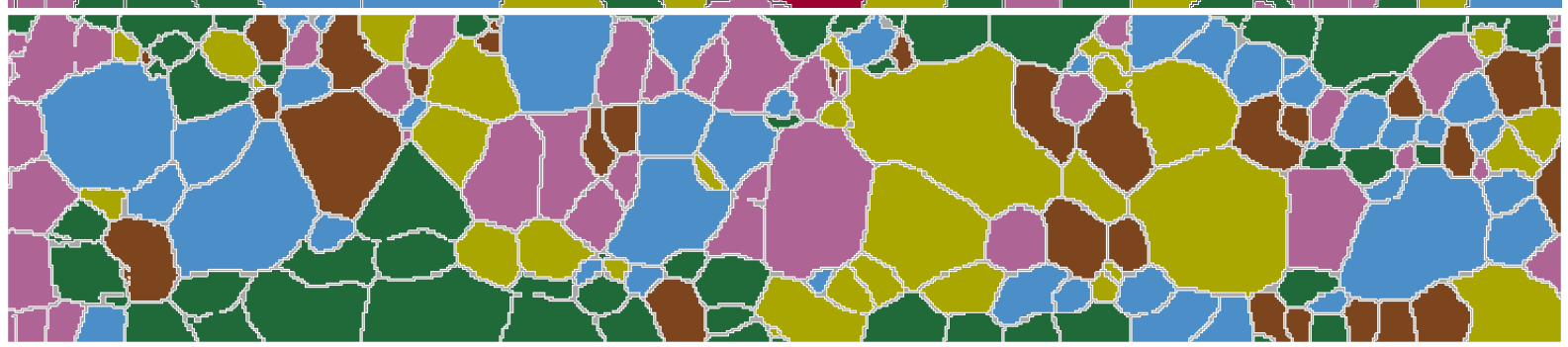

Figure 2. (a) Grain area distribution, (b) grain geometrical orientation distribution, (c) simulation model based on grain areas, and (d) simulation model based on grain orientations. 
If the microstructural information is difficult to obtain for some tiny samples, such as in-plane microstructures of ultra-thin sheets used for micro sheet forming, the Voronoi structures can also be used via the first modelling method. The grains and grain boundaries can also be considered separately. The grains areas and orientations can be computed and used for classification of the Voronoi cells. Fig. 3(a) illustrates a centroid Voronoi structure, Fig. 3(b) the detected grains from the Voronoi structure and Fig. 3(c) its corresponding simulation model considering both grains and grain boundaries. (a)

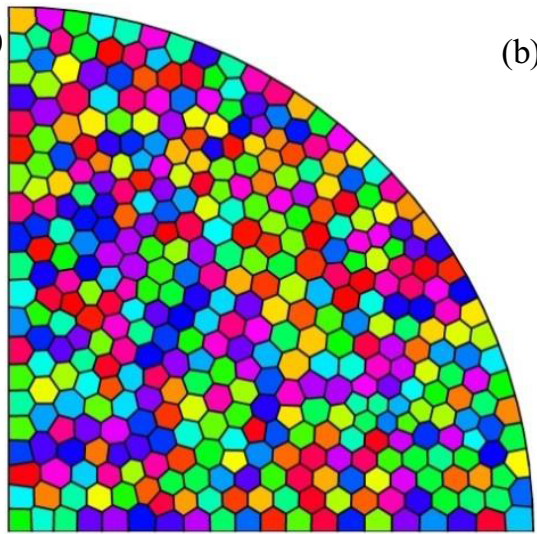

b)

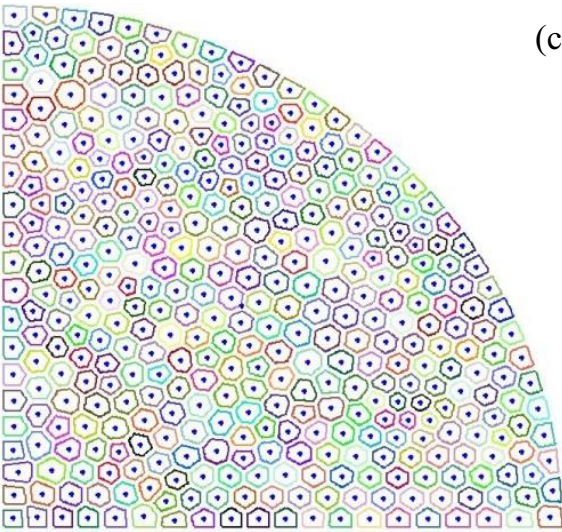

(c)

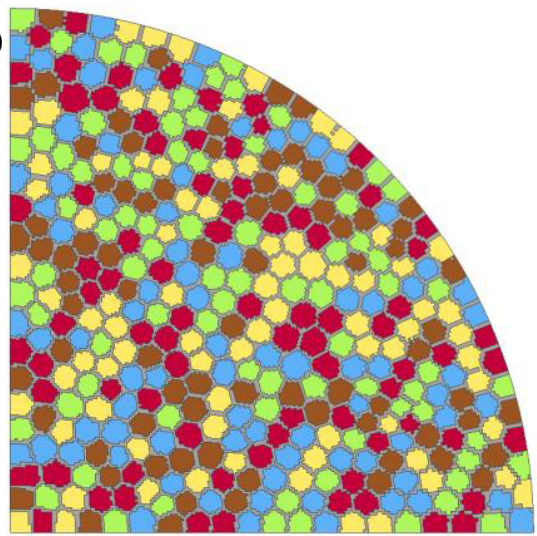

Figure 3. (a) Centroidal Voronoi structure, (b) detected grains and (c) corresponding simulation model from the first modelling method.

\section{Modelling method II}

There is another method to generate the real microstructure model from colourful or grey level images. If the images are coloured by the orientation or the Eulerian angles, the second modelling method can be used to build the real microstructural simulation model. Fig. 4(a) exhibits the orientation information obtained from EBSD where the colour indicates different orientations of the grains. Unlike the first modelling method, the colourful image was read into MATLAB without binarisation process. The colour information was discretised into the red, green and blue (RGB) system and kept as a 3D matrix composited by three 2D matrixes. Moreover, every pixel on the image has the red, green and blue values ranging from zero to 255 from the three $2 \mathrm{D}$ matrixes respectively. The position of a data point in the 2D matrix is the pixel positon on the image. Therefore, the pre-built mesh was mapped onto the colourful image, and each element was projected to a pixel according to their central coordinates. Similarly, all the grains were arranged as several groups with individual mechanical properties based on the RGB colour values. As the grain boundaries on the image were of black, the elements being of black were defined as the grain boundaries. Fig. 4(b) shows the developed simulation model based on the colourful image including the orientation information. Therefore, the real microstructural simulation model considering grains and their boundaries respectively can be generated. If grey level images were used, the RGB values were replaced by the grey level value ranging from zero to 255 too. Similarly, all the elements were arranged according to the grey level values. As the colour information indicates orientation, grains with close colour were naturally defined as one part with a type of mechanical properties. The grain boundaries were also set the average mechanical properties of all grain groups.

(a)

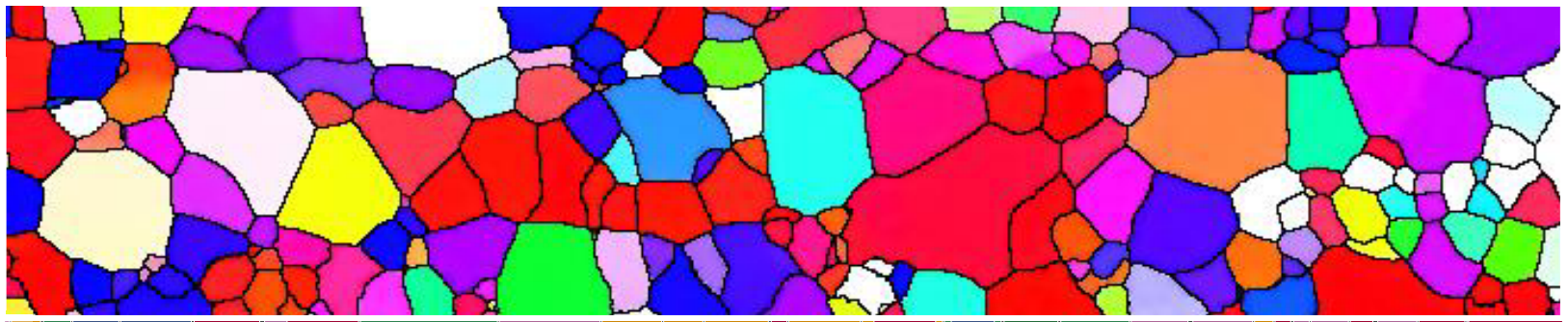

(b)

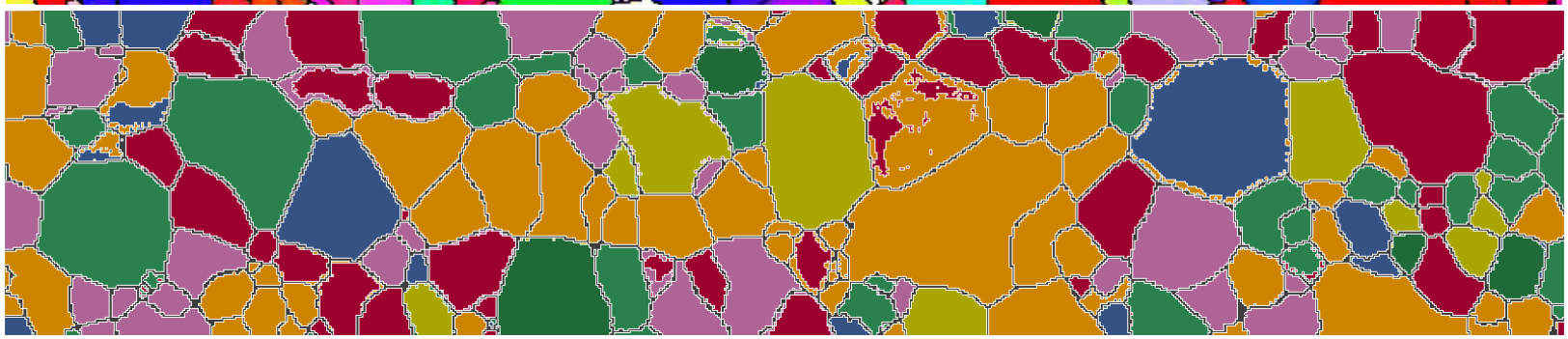

Figure 4. (a) Orientation image from EBSD and (b) its corresponding simulation model. 
If the microstructural information is impossible, the Voronoi structures can also be utilised in the second modelling method. Fig. 5(a) shows the centroidal Voronoi structure representing the annealed metal with

(a)

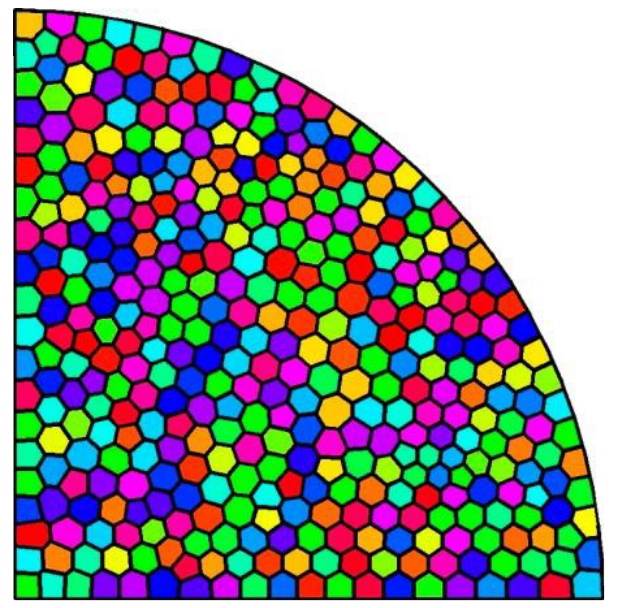

equiaxed crystals, and Fig. 5(b) displays its FE simulation model. All the grains were classified based on the Voronoi cell colours and grain boundaries were identified by black. (b)

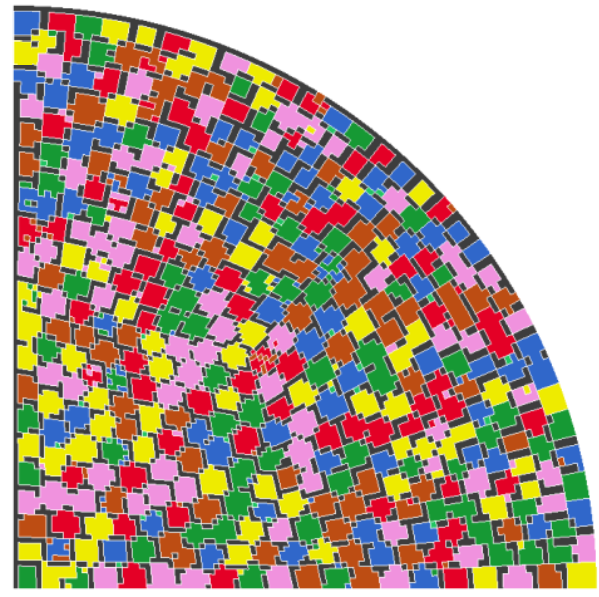

Figure 5. (a) Centroidal Voronoi and (b) its FE simulation model from the second modelling method.

\section{Discussion}

The mesh of the new model is independent on the images or the Voronoi structures for both two modelling methods. Thus, the total simulation model size can be small and executable for the commercial software while the mesh quality can be kept at a high level. However, it is better to generate the mesh based on the grains and their boundary sizes. With relatively large elemental sizes compared to the grain sizes, the grains cannot be presented smoothly and the grain boundaries might be lost.

Regarding the first modelling method, all the grains are detected as the closed subareas. Therefore, grain geometrical information, such as the area and geometrical orientation, can be obtained and used for further sorting of the grains. However, for the ultra-small grains on a low-resolution image, the detected grains may be of lines or curves but not closed geometries due to the boundary searching algorithm applied in the program. Although this information can be used, it is generally deleted as noise.

The second modelling method links the element to its corresponding RGB or grey level values. Therefore, the meaning of the colour values is included in the model naturally. Moreover, the grains are grouped automatically and grain boundaries can be found as they have fixed colour value (normally black). Due to the colour transition and limited number of grain groups, it is possible to arrange the elements belonging to one grain in different grain groups.

To validate this new model, micro deep drawing models were built and run. The Voronoi structure was utilised and both grains and their boundaries were considered in the new model. As the Voronoi structure was used, the new models built by the two modelling methods were almost the same, except the grain group distribution. Fig. 6(a) and (b) display the blank models from the first and the second modelling methods respectively, Fig. 6(c) and (d) show the effective stress of the drawn cups using the first and second blank models with an average grain size of $40 \mu \mathrm{m}$ respectively. Localised deformation and material inhomogeneity can be reflected by the new models. Therefore, the stress is uneven at the same height level on the drawn cup, as shown in Fig. 6(c) and (d).

A Voronoi model without consideration of grain boundaries and a normal deep drawing model were also developed for comparison. In addition, the blanks with thickness of $50 \mu \mathrm{m}$ were annealed at $1100{ }^{\circ} \mathrm{C}$ and drawn into micro cups. The microstructure of the blank was of equiaxed crystals and the average grain size was $40 \mu \mathrm{m}$. Fig. 7(a) displays the mouth of the drawn cup and Fig. 7(b-d) exhibit thickness distribution of the drawn cups mouths from the developed new model, a Voronoi model without consideration of grain boundaries and a normal model without account of material inhomogeneity, respectively. The maximum wall thickness at the cup mouth area was selected for comparison between the simulation and the experimental results. Without consideration of the material microscopic heterogeneity, localised deformation cannot be presented and therefore the maximum thickness was averaged resulting in the lowest peak thickness values among all the simulation. By contrast, the new model and the Voronoi model considered microscopic heterogeneity. Higher maximum thickness than that in the normal model was obtained from these two models. Furthermore, without buffer of the grain boundaries, the Voronoi model presented the largest thickness among all simulations. Fig. 8 compares the maximum thickness between both simulation and average experimental results. The developed model presented closer results to the experimental results than the other simulation models. 
(a)

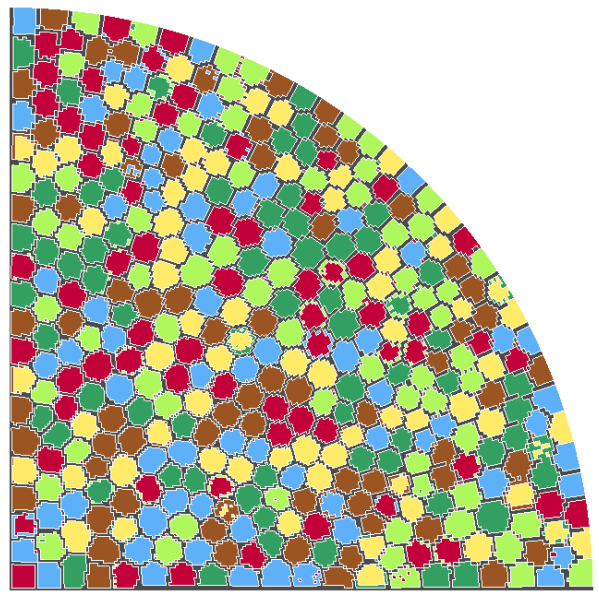

(c)

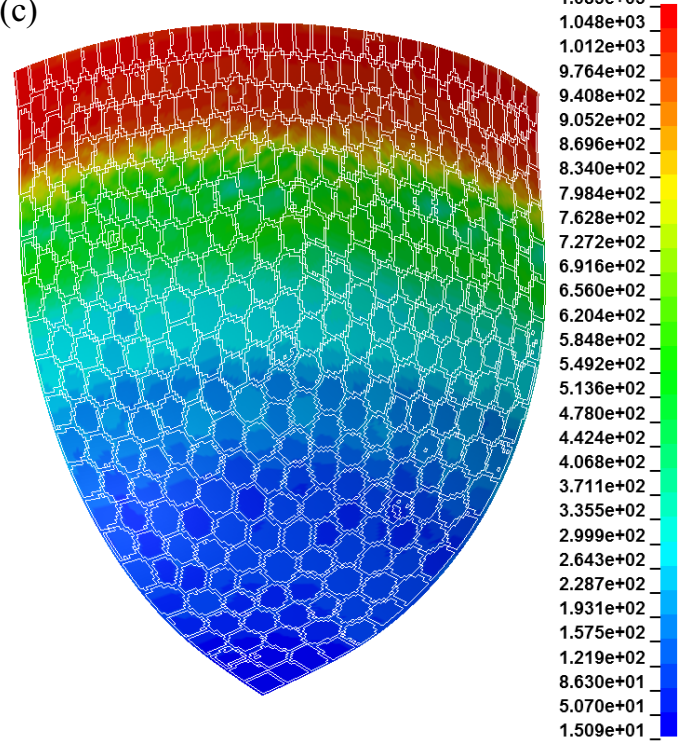

(b)

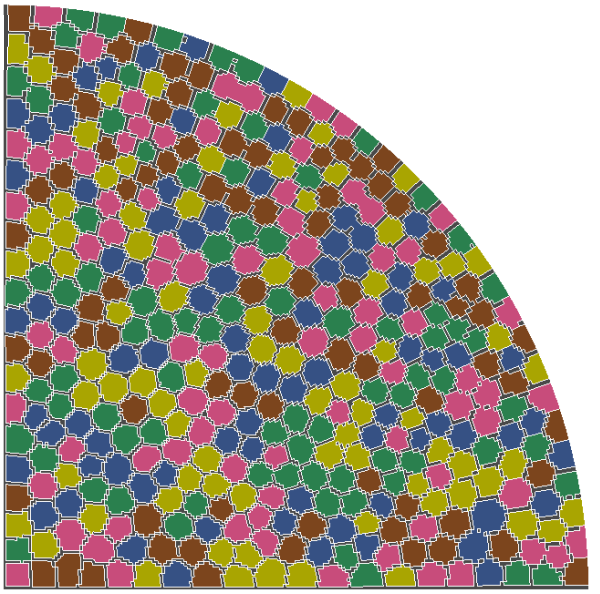

(d)

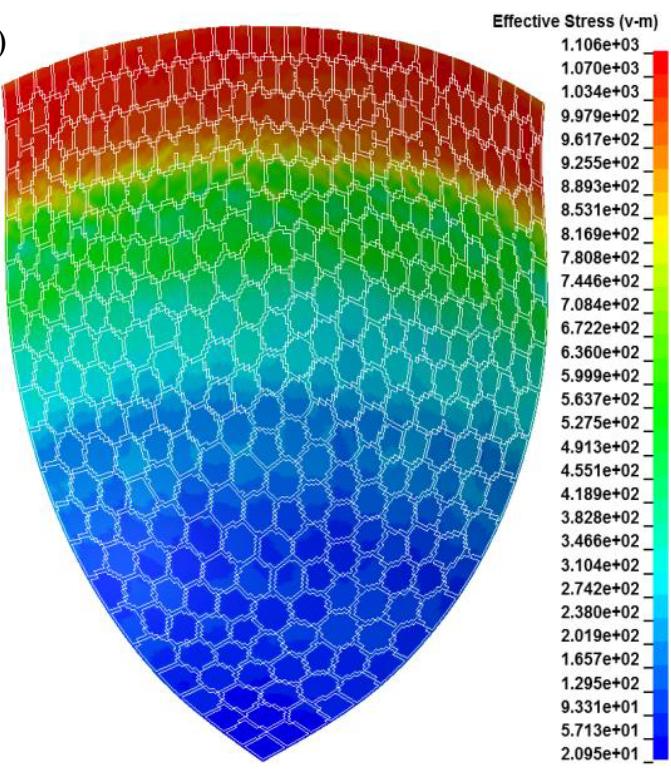

Figure 6. (a), (b) Blank models from the first and second modelling methods respectively and (c), (d) effective stress of the drawn cup from the first and the second models.

\section{Conclusions}

The micro scale simulation model considering both grains and their boundaries can be developed successfully through the two modelling methods. Moreover, the two modelling methods can include the primary microstructural information.

The first method utilises various microstructural information with clear boundaries, such as the images from the metallurgic microscope and EBSD, to detect the grains and then the grain boundaries. The grain sizes and geometrical orientations can be calculated and used for classification of grains. Therefore, the grains can be reasonably categorised based on these geometrical information. Furthermore, the Voronoi structures can also be applied via this modelling method, and therefore grain boundaries can be considered. The second modelling method utilised the colour information of the image to detect grain boundaries and classify grains naturally. Similarly, the Voronoi structures can be applied via the second method, if the real microstructure is difficult to obtain.

The developed new model via either of the two modelling methods can present the samples on the grain level considering grains and grain boundaries individually. Therefore, the size effects, especially the localised deformation and influences of the individual grain, can be well presented. The developed new model provides closer results to the experimental results, regarding the maximum thickness, than the Voronoi model and the normal model. This indicates the importance of consideration of grain boundaries in micro FE simulation. 

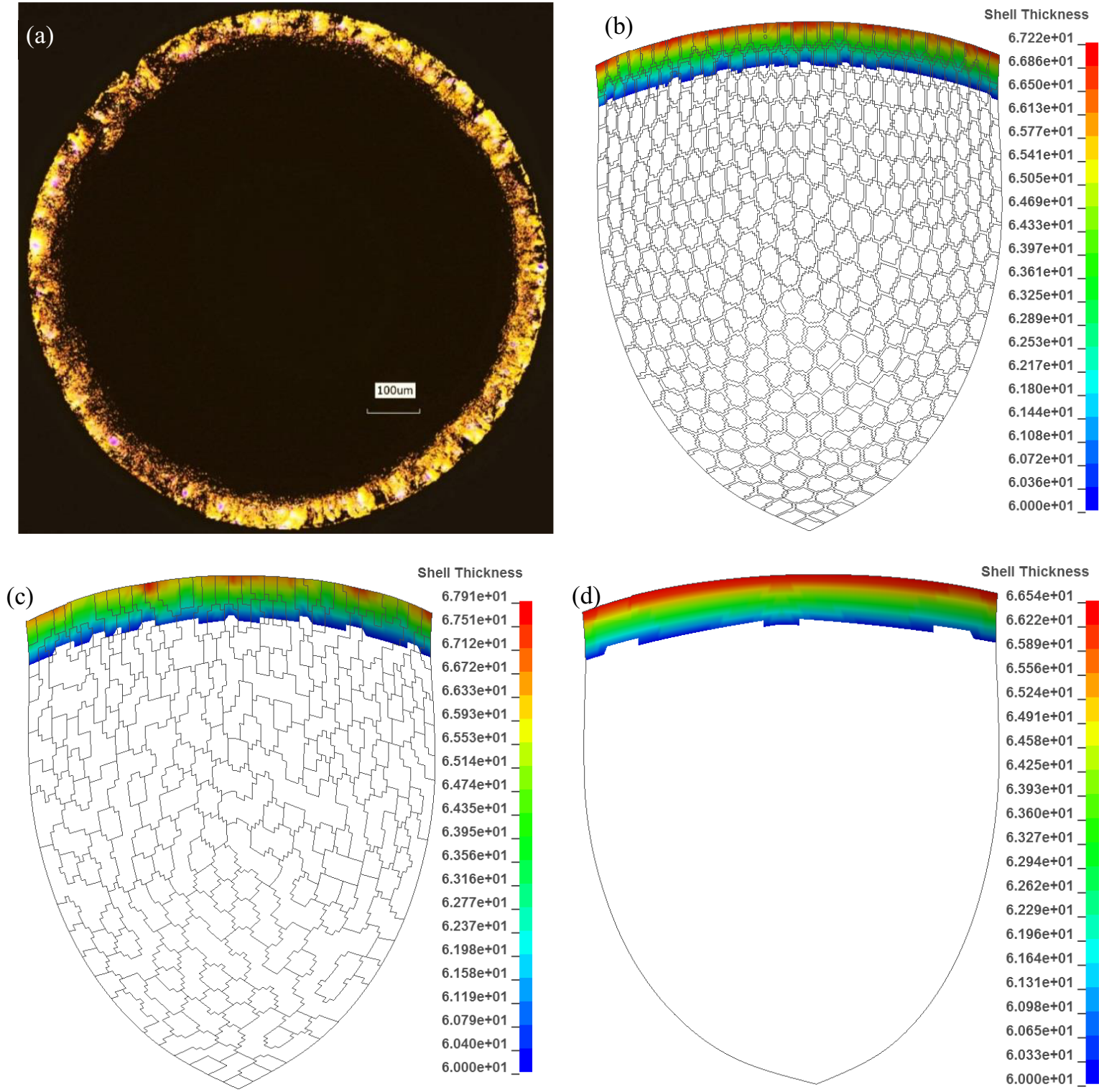

Figure 7. (a) Drawn cup with $1100{ }^{\circ} \mathrm{C}$ annealed blank and maximum thickness distribution from the simulation with (b) the developed new model, (c) the Voronoi model and (d) the normal model.

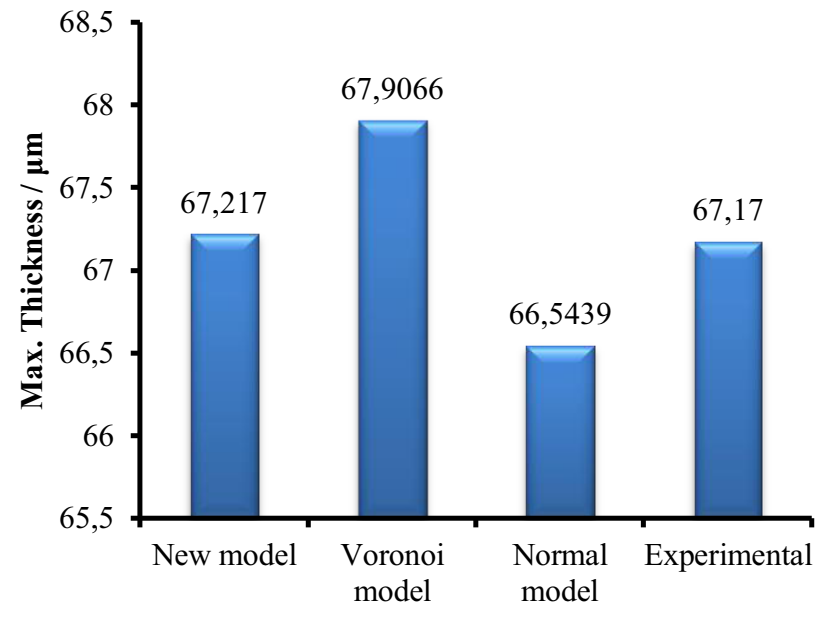

Figure 8. Comparison of the maximum thickness among different simulation models and average experimental results.

\section{Acknowledgements}

The first author greatly thanks for the finical support from the Chinese Scholar Council (CSC 201206160011) and the International Postgraduate Tuition Award (IPTA) offered by the University of Wollongong. This work is also supported by Australian Research Council (ARC) for Future Fellowship, and Open Research Fund from the Sate Key Laboratory of Rolling and Automation, Northeastern University, China (Contract No.: 2014003): The authors also thank that Mr. Guoqing $\mathrm{Zu}$ provided the experimental images of Figs. 1(a) and 4(a).

\section{References}

1. Z. Xu, L. Peng, X. Lai, M. Fu, Mater. Sci. and Eng.: A. $611345-353$ (2014)

2. A. Molotnikov, R. Lapovok, C.F. Gu, C.H.J. Davies, Y. Estrin, Mater. Sci. and Eng.: A. 550 312-319 


\section{(2012)}

3. F. Vollertsen, Key Eng. Mater. 473 3-12 (2011)

4. G.C. Wang, W. Zheng, T. Wu, H. Jiang, G.Q. Zhao, D.B. Wei, Z.Y. Jiang, J. Mater. Process Technol. 212 678-684 (2012)

5. F.H. Yeh, C.L. Li, Y.H. Lu, J. Mater. Process Technol. 201 237-241 (2008)

6. N. Ma, Huazhong Univ. of Sci. and Technol. (2005)

7. N. Ma, X.H. Dong, J. Shanghai Jiaotong Univ. 12.3
323-327 (2007)

8. L. Luo, Z.Y. Jiang, H.N. Lu, D.B. Wei, K.Z. Linghu, X.M. Zhao, D. Wu, Procedia Eng. 81 2409-2414 (2014)

9. H.N. Lu, D.B. Wei, Z.Y. Jiang, X.H. Liu, K. Manabe, Comp. Mater. Sci. 77 44-52 (2013)

10. C.A. Sweeney, P.E. McHugh, J.P. McGarry, S.B. Leen, Int. J. Fatigue 44 202-216 (2012) 